\title{
Glaube und Vernunft. Die Trinitätstheologie des Albertus Magnus
}

Die mittelalterliche Theologie verstand sich als die geistige Durchdringung und die systematische Darstellung der im Glauben bejahten Offenbarung. Das ist eine unbestreitbare Tatsache. Aber diese Gegebenheit besagt an und für sich sehr wenig. Weit wichtiger ist die Frage, wie diese Durchdringung und Systematisierung geschah und welche methodologischen und philosophischen Mittel dabei eingesetzt wurden. Hier traten große Unterschiede auf. Ein kurzer Blick in die Geschichte genügt, um die Verschiedenheit der Erklärungsversuche festzustellen: Die Methode des Alkuin ist eine andere als die des Anselm, und die Schriften des Bonaventura atmen einen anderen Geist als die des Thomas von Aquin.

Die in diesen methodischen Bemühungen bezogenen Positionen besagen etwas über die Rolle und Bedeutung, die der menschlichen Vernunft in der Erschließung der göttlichen Wahrheit zuerkannt wurde. Eine Untersuchung der verschiedenen Positionen ist deshalb nicht nur theologisch interessant, sondern hat auch eine direkte Relevanz für die Geschichte der Philosophie, vor allem wenn es um die rationale Erklärung fundamentaler Wahrheiten wie der Trinität und der Inkarnation geht, die als unergründliche Geheimnisse des Glaubens galten. Hier stößt die Philosophie an ihre Grenze und das von ihr verteidigte Konzept der Rationalität steht zur Diskussion ${ }^{1}$.

Albertus Magnus ist im Hinblick auf diese Problematik eine interessante Persönlichkeit. Er zeigt ein deutliches Bewußtsein davon, daß die Anwendung philosophischer Begriffe in der Theologie zu Widersprüchen führen kann. In der Natur sind alle Prozesse gekennzeichnet durch das Zusammenspiel von Wirkung und Erleiden, auch beim Hervorbringen und Zeugen. Aber in Gott gibt es kein Erleiden. Es scheint also unmöglich, die innergöttliche Hervorbringung des Sohnes durch den Vater philosophisch zu erklären. Dennoch lehrt der Glaube, daß der Sohn vom Vater gezeugt ist. Die Sprache der Philosophie, wenn sie sich an die natürliche Bedeutung der Begriffe hält, scheint also für die geistige Durchdringung der Trinität ungeeignet ${ }^{2}$.

Dieser Umstand bedeutet für Albertus jedoch nicht das Scheitern der Philosophie in ihrer Anwendung in der Theologie. Er betrachtet ihn eher als eine Beschränkung der in der Naturphilosophie gängigen Bedeutungen der Begriffe. Die Theologie ist sehr wohl imstande, die Trinität mit philosophischen Mitteln begrifflich zu erschließen, auch wenn sie hier keinen absoluten Beweis liefern kann. Die befolgte Methode sollte dann 
jedoch dem Gegenstand angepaßt sein $^{3}$. Da Gott hier der Gegenstand ist, wird eine besondere Methode verlangt, die der göttlichen Natur in ihrer dreipersönlichen Wirklichkeit eigens Rechnung tragen kann. Diese Natur wird am besten dadurch zum Ausdruck gebracht, daß Gott das höchste Gut ist. Das Ziel unserer Untersuchung ist es nun, diese Methode in ihrer Anwendung auf das höchste Gut herauszuarbeiten.

Wir möchten mit einer Erörterung der Theologie als Wissenschaft beginnen, wie Albertus sie durchgeführt hat. Damit wird der Rahmen abgesteckt, innerhalb dessen seine Trinitätslehre verstanden werden soll. Kennt man das von Albertus bestimmte Ziel der Theologie, so lassen sich die spekulativen Theorien beurteilen und es wird deutlich, wo die Akzente in der Darstellung der Trinität zu suchen sind. Anschließend gehen wir dann auf die Trinitätslehre und die dort angewandte Methodik selbst ein. Drei Aspekte werden dabei im Mittelpunkt stehen: die innergöttliche Zeugung des Sohnes, die innergöttliche Beziehung zwischen Einheit der Natur und Vielheit der Personen und die Frage, weshalb es nur drei und nicht mehr göttliche Personen gibt. Zum Schluß wenden wir uns dann der Rezeption der Methodik Alberts bei Heymericus de Campo und Nicolaus von Kues zu.

\section{Natur und Gegenstand der Theologie}

Albertus betrachtet die Theologie als höchste Form des Wissens. Sie ist die sicherste Wissenschaft, denn sie ist im ersten Prinzip der Wirklichkeit begründet: in Gott selbst. Gott ist unveränderlich und vollkommen erkennend. Sein Wesen ist die höchste Form der Vernünftigkeit. Diese Vernünftigkeit ist Prinzip und Ursache jeder anderen Form der Vernünftigkeit, auch der des Menschen. Im wissenschaftlichen Erkennen partizipiert der Mensch demnach an der göttlichen Vernunft. In der Theologie geschieht das in der höchsten Form, die dem Menschen möglich ist. Denn dort ist Gott sowohl Prinzip als auch Ziel des Erkennens ${ }^{4}$.

Diese partizipative Form des Erkennens wird von Albertus mit dem Begriff der Abbildung (sigillatio) zum Ausdruck gebracht. Der menschliche Geist ist ein Abdruck der göttlichen Weisheit, gestaltet nach der Form und den Prinzipien (rationes) der ersten Ursache. Durch diese Beziehung hat der menschliche Geist eine natürliche Neigung zur göttlichen Weisheit. Insofern ist die Theologie eine Wissenschaft, die zur Natur des Menschen gehört. Aber der Mensch kann diese Weisheit nicht selbst erreichen. Es muß von Gott geholfen werden. Deshalb ist in der Theologie nicht nur das wissenschaftliche

Ebd.

ALBERTUS Magnus: Summa I, prol.: Ed. Colon. t. 34,1, S. 1a-4b. 
Forschen wichtig, sondern auch das Gebet und die Hingabe. Das Studium disponiert zur Weisheit. Aber erst durch das Gebet und die Hingabe kann der Mensch zu Gott emporsteigen ${ }^{5}$.

Trotz allen Strebens bleibt die menschliche Erkenntnis Gottes immer beschränkt. Der Grund dafür liegt nicht so sehr in der Verbindung der menschlichen Seele mit dem Körper (wie das nach Thomas der Fall ist), sondern in der göttlichen Unendlichkeit. Der Mensch kann in seinem Denken von Raum und Zeit abstrahieren und so zum Bereich der reinen Vernünftigkeit emporsteigen. Aber er wird die göttliche Wesenheit niemals erfassen können. Hier stößt er an eine absolute Grenze: die göttliche Unendlichkeit. Gott ist ohne Grenze. Er wird durch nichts eingeschränkt oder in seiner Wesenheit bestimmt. Das menschliche Denken kann Gott also nicht in einem Begriff erfassen, sondern seine Wesenheit nur streifen. Damit zeigt sich eine besondere Dynamik der Theologie, durch die sie sich von den anderen Wissenschaften unterscheidet. In der Theologie kann der Mensch dem Gegenstand immer näher kommen, aber er wird ihn niemals erreichen. Das ist keine Armut, sondern Zeichen der Vollkommenheit des Gegenstandes ${ }^{6}$.

Die Unendlichkeit Gottes wird von Albertus mit dem Begriff des höchsten Gutes (summum bonum) zum Ausdruck gebracht. Die göttliche Wesenheit umfaßt alle Vollkommenheiten in größter Erhabenheit. Ihr kann nichts hinzugefügt werden. Nichts kann ausgedacht werden, das in irgendeiner Weise vollkommener ist als die göttliche Wesenheit. Die göttliche Vollkommenheit ist in jeder Hinsicht absolut (substantialiter et essentialiter). Deshalb ist sie unbeschränkt und deshalb setzt Gott selbst sein eigenes Maß und Ziel (ipse se finit) ${ }^{7}$.

In den Geschöpfen, die an der göttlichen Vollkommenheit teilhaben, fehlt diese Absolutheit. Die geschöpflichen Vollkommenheiten sind ihrem Wesen nach begrenzt und damit auch voneinander unterschieden. Im Bereich des Geschöpflichen lassen sich Vollkommenheiten finden oder ausdenken, die in einem höheren oder niedrigeren Grade vollkommen sind als andere ${ }^{8}$. Die Schöpfung hat kein absolutes Maß in sich, sondern nur in Gott, dem höchsten Gut. Das trifft auf eine besondere Weise auf die Theologie zu, wenn der Mensch als Geschöpf von Gott dem dreieinigen Schöpfer spricht.

Die Theologie spricht von Gott nach seiner ganzen Vollkommenheit. Gott wird hier also nicht nur als erste Ursache betrachtet, sondern auch als letztes Ziel. Albertus hebt ausdrücklich hervor, daß eine Theologie, die Gott nur nach seiner Substanz betrachtet, nicht vollständig ist. Gott ist Ursprung und Ende der Schöpfung und nur wenn er nach der Ganzheit seines Wesens betrachtet wird, so daß aus diesem Wesen die Schöpfung

Ebd.: S. 1b.

Ebd.: S. 3b.

ALBERTUS Magnus: I Sent., d. 2, a. 2: Ed. Paris. t. 25, S. 56b-57b.

Ebd. 
ausströmt und zu diesem Wesen die Schöpfung zurückkehrt, wird er in seiner Vollkommenheit verstanden. Die Theologie handelt also nicht so sehr von Gott als dem Wahren schlechthin (verum simpliciter), sondern eher von Gott als dem höchsten Glücksziel der Schöpfung (summe beatificans) ${ }^{9}$.

Die Theologie hat deshalb eine unverkennbar praktische Seite. Sie betrifft die ganze Schöpfung und den ganzen Menschen in seinem Streben nach Gott. Dieses Streben ist beim Menschen nicht nur intellektuell, sondern ist mit einer devotionalen Hingabe eng verbunden. Gott ist zwar die höchste Form der Vernünftigkeit, aber durch seine Unendlichkeit für den Menschen nicht vollständig erkennbar. Er bleibt für die menschliche Vernunft zum Teil verborgen. Den Abstand zum Verborgenen Gottes kann nicht durch die Vernunft, sondern nur durch die Hingabe an Gott überbrückt werden. Theologie ist nach Albertus dann auch wesentlich eine scientia affectiva. Sie ist praktisch und devotional nicht nur insofern sie die Verehrung Gottes zum Gegenstand hat, sondern auch und vor allem weil sie den Menschen in seinem moralischen Leben auf Gott ausrichtet $^{10}$.

Dieser Begriff der Theologie ist für unser Verständnis der Trinitätslehre Alberts wichtig und zwar aus zwei Gründen.

Erstens ist die Erörterung der Trinität nach Albertus offensichtlich keine selbständige Studie des göttlichen Wesens, sondern steht in direktem Zusammenhang mit der Theologie als Lehre des menschlichen Heils. Die Selbsterschließung Gottes ist Ursprung und Ziel der göttlichen Heilsgeschichte. Erst auf dem Hintergrund dieser Heilsgeschichte gewinnt die Trinität ihre eigentliche Bedeutung für den Menschen, der die göttliche Natur mit der Vernunft zu ergründen sucht ${ }^{11}$.

Zweitens erschließt der Mensch in der Erörterung der Trinität nicht nur die innere Struktur der göttlichen Vernünftigkeit, sondern es wird ihm auch deutlich, wie das Erkennen dieser höchsten Form des Wissens, die für ihn Ziel der Glückseligkeit ist, sich im praktischen Vollzug gestaltet. In der Betrachtung der Trinität ist der Mensch auf die göttliche Offenbarung angewiesen. Den Inhalt dieser Offenbarung kann er nur in einem freien Akt des Willens annehmen und ihr zustimmen. Der Wille und der Intellekt ste-

I Sent., d. 1, art. 2-3: Ed. Paris. t. 25, S. 16a-17b; Summa I, tr. 1, q. 2: Ed. Colon. t. 34,1, S. 8a-9b u. ebd.: q. 3 c. 3 : S. 13 a-b.

ALBERTUS Magnus: Summa I, tr. 1, q. 3 c. 3: Ed. Colon. t. 34,1, S. 13a-b. Dazu ausführlich SENNER, Walter: Zur Wissenschaftstheorie der Theologie im Sentenzenkommentar Alberts des Großen. In: MEYER, Gerbert / ZIMMERMANN, Albert (Hrsg.): Albertus Magnus Doctor Universalis 1280/1980. Mainz 1980, S. 323-343; WÉBER, Édouard: La relation de la philosophie et de la théologie selon Albert le Grand. In: Archives de Philosophie 43 (1980), S. 559-588.

Das wird mit Blick auf die Schöpfung klar herausgearbeitet in EMERY, Gilles: La trinité créatrice. Trinité et création dans les commentaires aux Sentences de Thomas d'Aquin et ses précurseurs Albert le Grand et Bonaventure. Paris 1995 (Bibliothèque Thomiste, 47), bes. S. 39-161. 
hen in dieser hohen Form des Erkennens einander also nicht gegenüber, sondern ergänzen sich gegenseitig. Nur wenn der Mensch sich dem Wissen und dem Willen nach der göttlichen Betrachtung hingibt, findet er den Weg zur dreipersönlichen Wesensgestalt Gottes, die als eigentlicher Gegenstand der Theologie gilt.

\section{Die theologische Erörterung der Trinität}

Vergleicht man die Erörterung der Trinität mit den Betrachtungen über die Natur der Theologie, dann zeigt sich, daß Albertus in der Abhandlung der dort behandelten Fragen dem besonderen Charakter der Theologie deutlich Rechnung trägt. Beschränken wir uns auf die drei anfangs genannten Probleme, so tritt dieser besondere Charakter vor allem in der von Albertus befolgten Methode hervor, die man als das 'Maximalisieren' bezeichnen kann. Gott ist das höchste Gut (summum bonum). Bei Gott gibt es nichts, das ein Mehr oder Weniger zuläßt. Jede Vollkommenheit kommt ihm notwendig auf die bestmögliche Weise zu. Diese Strategie des Maximalisierens wird von Albertus auf Anselmus zurückgeführt und zwar in der Form der Regel: Alles Passende wird Gott notwendig zugesprochen und alles Unpassende notwendig abgesprochen (omne conveniens deo est necessarium et omne inconveniens impossibile $)^{12}$. Die beiden Teile dieser Regel, das Zuerkennen des Passenden und das Aberkennen des Unpassenden, gehören zusammen. In ihrer gegenseitigen Bestimmung machen sie die Dynamik der in der Regel dargelegten Methodik aus, die darin gipfelt, daß Gott nur das Allerhöchste zugesprochen werden kann. Alles, was weniger ist als das Beste, ist unpassend für Gott als summum bonum und kann ihm deshalb nicht zugesprochen werden.

Diese Strategie steht im Einklang mit der besonderen Natur der Theologie, denn sie bringt zwei entscheidende Aspekte zum Ausdruck, nämlich erstens, daß der Gegenstand der Theologie unendlich ist und deshalb vom Menschen niemals erfaßt werden kann, und zweitens, daß die Theologie ihren Gegenstand nicht a priori beweisen kann, sondern immer von der Schöpfung zu Gott aufsteigen muß. In der konkreten Durchführung sieht diese Strategie nämlich so aus, daß die Theologie die höchsten Vollkommenheiten in der von Gott hervorgebrachten Schöpfung sucht, sie von allem Unvollkommenen abstrahiert und sie dann in absoluter Form, die kein Mehr oder Weniger mehr zuläßt, von Gott aussagt. Sie ist damit eine Kombination der drei Wege zur Gotteserkenntnis: die via causalitatis, die via negationis und die via eminentiae. Obwohl Albertus diese Strategie, wenn er sie in der Trinitätslehre anwendet, immer mit Anselm

ALBERTUS Magnus: Summa I, tr. 7, q. 30, c. 1: Ed. Colon. t. 34,1, S. 229a. Diese Regel tritt auch an anderen Stellen hervor. Ihre Formulierung ist jedoch schwankend. Ähnliches kann auch bei anderen Autoren festgestellt werden, die in diesem Punkt auf Anselm verweisen, wie zum Beispiel in der Summa Halensis. 
verbindet, ist die eigentliche Quelle jedoch Pseudo-Dionysius. Das geht aus den Kommentaren Alberts zu Pseudo-Dionysius deutlich hervor. Dort legt Albertus die Grundlage dieser Methode und verbindet sie auch mit dem affektiven und praktischen Charakter der Theologie ${ }^{13}$.

\subsection{Die innergöttliche Zeugung des Sohnes}

Die von Albert verteidigte Lehre geht von der Interpretation der Trinität aus, wie sie in der allgemeinen IV. Kirchenversammlung im Lateran (1215) in der Auseinandersetzung mit Joachim von Fiore bestimmt wurde. Das Wesen Gottes ist vollkommen eins, zugleich aber drei Personen, die sich als Personen voneinander unterscheiden. Die drei Personen sind gleichen Wesens, gleicher Substanz und gleicher Vollkommenheit und mit dem göttlichen Wesen real identisch. Als Person jedoch haben sie jeweils andere Merkmale. Es ist der Vater, der zeugt, der Sohn, der gezeugt wird, und der Heilige Geist, der hervorgeht ${ }^{14}$.

Ist diese Lehre für den Theologen Ausgangspunkt seiner Betrachtungen, so ruft sie bei den Philosophen die Frage nach der Beziehung zwischen Einheit und Vielheit hervor. Die These, daß die göttliche Natur vollkommen eins ist und doch zugleich drei voneinander verschiedene Personen, scheint einen Widerspruch zu beinhalten. Einheit und Vielheit schließen sich aus und können nicht vom gleichen Gegenstand ausgesagt werden.

Dieses Problem hat Albertus an verschiedenen Stellen erörtert, am eingehendsten im Zusammenhang mit der Frage, wie der Vater den Sohn zeugen kann, ohne die Einheit des göttlichen Wesens zu gefährden. Dort trat Albertus auch mit den Philosophen in Diskussion, die behaupteten, jede Form des Zeugens setze eine Potentialität oder Bewegung beim Gezeugten voraus, was bei Gott unmöglich sei ${ }^{15}$.

Die von Albertus hier befolgte Argumentation ist ein Musterbeispiel der obengenannten Strategie des Maximalisierens. Zeugen (generare) heißt nach Albertus das Mitteilen der Natur (communicatio naturae). Je besser die Natur sich mitteilt, desto hoher ist der

Zur theologischen Methode Alberts siehe WÉBER, Édouard: L'interprétation par Albert le Grand de la Théologie mystique de Denys le Ps.-Aréopagite. In: MEYER, Gerbert / ZIMMERMANN, Albert (Hrsg.): Albertus Magnus Doctor Universalis 1280/1980. Mainz 1980, S. 409-439; RUELLO, Francis: Le commentaire du Divinis nominibus de Denys par Albert le Grand. In: $A r-$ chives de Philosophie 43 (1980), S. 589-613.

Vgl. DENZINGER, Heinrich / HÜNERMANN, Peter (Hrsg.): Kompendium der Glaubensbekenntnisse und kirchlichen Lehrentscheidungen. Freiburg i. Br. ${ }^{37} 1991$, n. 803-808, S. 359-362. Zur Trinitätslehre des Albertus mit ausführlichen Angaben zur weiteren Literatur siehe COURTH, Franz: Trinität in der Scholastik. Freiburg i. Br. 1985 (Handbuch der Dogmengeschichte, Bd. 2/1b) und EMERY [Anm. 11], S. 39-161.

ALBERTUS Magnus: I Sent., d. 4, a 3: Ed. Paris. t. 25, S. 159a-161a; Summa I, tr. 7, q. 30, c. 1: Ed. Colon. t. 34,1, S. 226a-229a. 
Grad des Zeugens. Die Aufgabe, die Albertus sich hier dann auch stellt, ist es, den höchsten Grad des Zeugens zu bestimmen ${ }^{16}$.

Bei den körperlichen Wesen ist der Prozeß des Zeugens immer mangelhaft, da er, wie die Philosophen zu Recht behaupten, Potentialität und Bewegung beim Gezeugten voraussetzt und Zeugender und Gezeugtes deshalb immer substantiell getrennt sind. Die Übereinstimmung zwischen Zeugendem und Gezeugtem ist nur partiell und somit auch die Mitteilung der Natur ${ }^{17}$.

Steigt man auf zum Bereich des Denkens, dann gewinnt das Zeugen an Vollkommenheit. Es kommt zum absoluten Höhepunkt im göttlichen Denken, wenn das Denken etwas von ihm Gedachtes hervorbringt, das gleicher Natur und gleicher Substanz ist. Bringt das göttliche Denken ein Substantielles von ihm Gedachtes hervor, so sind beide notwendig wesensgleich, ungeachtet des Unterschieds zwischen Denken und Gedachtem ${ }^{18}$.

Dies ist nach Albertus die höchste Form des Zeugens. Sie stellt die oberste Stufe dar, denn die Mitteilung der Natur ist im göttlichen Denken maximal und somit auch substantiell ${ }^{19}$. Denken und Gedachtes sind folglich einer Natur. Diese Form kann Gott zugesprochen werden, denn Gott ist als allgemein tätiger Intellekt (intellectus universaliter agens) die höchste Form des vernünftigen Lebens ${ }^{20}$. Sie übersteigt das menschliche Erkennen. Denn die Natur des denkenden Menschen und das von ihm Gedachte sind nicht wesensgleich. Beim Menschen ist das Gedachte nur ein Abbild des Erkannten und somit nicht substantiell. Zudem ist der Mensch im Erkennen vom Erkannten abhängig. Er kann den Inhalt seiner Erkenntnisbilder nicht selbst hervorbringen, sondern ist auf Sinnesdaten angewiesen. Bei der ersten Ursache kann man eine derartige Abhängigkeit jedoch nicht annehmen. Sie denkt ihre eigene denkende Natur. Hier sind Denken und Gedachtes der Natur nach wesensgleich und substantiell und man kann den absoluten Grad des Zeugens notwendig voraussetzten ${ }^{21}$.

\section{Ebd.}

Ebd.

18 Vgl. ALBERTUS Magnus: Summa I, tr. 7, q. 30, c. 1, sol.: Ed. Colon. t. 34,1, S. 228, 71-75: „Non enim negari potest, quin formatum ex intellectu distinctum sit ab ipso relatione originis; et cum non formetur nisi ex ipso, negari non potest, quin sit coessentiale et indistans ab ipso“. Siehe auch ebd.: q. 31, c. 2: S. 244b (5). Op. cit., q. 30, c. 2: S. 230a.

Op. cit., q. 30, c. 1, sol.: S. 228, 79-81: „Nobilissima enim vita est intelligere et maxime intelligere secundum universaliter agentem intellectum".

Vgl. op. cit., q. 30, c. 1 ad 6-12: S. 229, 6-10: „Quod enim in divinis sit generatio et perfectissima et prima, concedendum est, et rationes ad hoc inductae procedunt de necessitate eo modo quo dicit Anselmus, quod omne conveniens deo est necessarium et omne inconveniens impossibile“. 
Bei dem absoluten Grad gibt es somit ein Zusammenfallen von Einheit und Vielheit. Die Einheit der Natur geht mit dem Unterschied zwischen Zeugendem und Gezeugtem zusammen. Das ist zugleich die höchste Form der Vernünftigkeit und sie gehört auch zur höchsten Form der Ursächlichkeit. Gott ist das höchste Gut und in ihm wird das Gezeugte auf vollkommenste Weise gezeugt. Es gibt nichts im Gezeugten, das nicht auch im Zeugenden ist. Beide haben die gleiche Natur und sind von gleicher Vollkommenheit. Die Einheit der göttlichen Natur bringt sich in der Gleichheit mit sich selbst hervor $^{22}$.

Als höchste Form des substantiellen Zeugens stellt die Gleichheit mit sich selbst das Maß für jede andere Art des Zeugens oder Hervorbringens dar. Die Philosophen sind nach Albertus denn auch nicht konsequent, wenn sie nur an den niedrigen Formen des Zeugens festhalten (die in der Tat auf Gott nicht zutreffen). Das Niedrigere gründet im Höheren. Die Trennung von Zeugender und Erzeugtem in der Natur setzt so die Einheit von Zeugender und Erzeugtem im göttlichen Denken voraus. Die Position der genannten Philosophen ist deshalb nicht haltbar und leidet an einer inneren Inkonsistenz ${ }^{23}$.

Nach Albertus handelt die Theologie von Gott als Ursprung und Ziel der Schöpfung. Das trifft auch für die Analyse des göttlichen Zeugens zu. Das Gezeugte ist nicht nur wesensgleich mit dem Zeugender, sondern auch Urbild für alles andere, was von Gott hervorgebracht werden kann. Als solches stellt das in Gott Gezeugte den Archetyp der Schöpfung dar. In ihm existiert die höchste Form des Lebens, die von Albertus als tätiges Erkennen gedeutet wird. In Gott als intellectus universaliter agens findet jede Form des Lebens ihr eigenes Ziel. Die Dynamik der göttlichen Trinität ist somit Grund und Urbild des geschöpflichen Lebens ${ }^{24}$.

\subsection{Einheit der Natur und Vielheit der Personen}

$\mathrm{Daß}$ Gott seine Natur im Erkennen mitteilt, gilt nicht nur beim substantiellen Erzeugen des Sohnes sondern auch beim Hervorbringen des Heiligen Geistes. In beiden Fällen geht es um die gleiche göttliche Natur, die in jeder Hinsicht identisch mit sich bleibt wegen der vollkommenen göttlichen Einheit. Warum in diesem Denken des Denkens die göttliche Natur sich in zwei und nicht in nur einer oder sogar in mehreren Personen ausdrückt, wird weiter unten zu untersuchen sein. Jetzt gilt es, der Frage nachzugehen, wie Albertus die Beziehung zwischen der Einheit der göttlichen Natur und den Unterschieden zwischen den göttlichen Personen deutet.

Vgl. op. cit., q. 30 , c. 3 , a. 1 , sol.: S. $233,33-35$ : , ... in divinis, ubi maxima simplicitas est, necesse est unam re et numero esse essentiam genitoris et geniti sive patris et filii“". 
Ausgangspunkt ist für ihn die im Glauben festgehaltene Offenbarung, die göttliche Natur sei eins, die Personen jedoch verschieden. Diese Wahrheit versucht er nun im Rahmen seiner Theologie, die Gott als höchste Form der Vernünftigkeit betrachtet, dem menschlichen Verstand näher zu bringen. Seine Aṇtwort läßt sich wie folgt auf den Punkt bringen: Die göttliche Vernünftigkeit gestaltet sich in ihrer natürlichen Tätigkeit derart, daß Einheit und Vielheit notwendig zusammengehen müssen. Die Einheit ist der Grund des Vermögens, wohingegen die Vielheit die Tätigkeit des Vermögens zum Ausdruck bringt. Dem Wesen und der Vollkommenheit nach sind Grund und Tätigkeit des Vermögens von einer Substanz und einer Natur. Dennoch können den Tätigkeiten Eigenschaften zugesprochen werden, die der Natur als Grund nicht zukommen. So gehört es zur göttlichen Natur als höchster Vernunft, sich im Gedachten auszudrücken. Drückt sie sich aber im Gedachten aus, so entsteht trotz der Einheit der Natur ein notwendiger Unterschied zwischen dem Denken als Zeugender und dem Gedachtem als Erzeugtem. Dieser Unterschied betrifft nicht die Natur oder die Substanz. Das göttliche Wesen bleibt vollkommen ungeteilt. Der Unterschied bezieht sich eher auf die Art und Weise, wie die Natur sich in ihrer Tätigkeit tatsächlich mitteilt. Hier gibt es Beziehungen, die sich gegenseitig ausschließen. Denken ist nicht Gedachtes und Gedachtes ist nicht Denken ${ }^{25}$.

Albertus verwendet einige technische Begriffe, um den besonderen Umstand zum Ausdruck zu bringen, nach dem die Einheit in der Natur und der Unterschied in den Personen besteht. Die Vernunft Gottes ist wegen der Einheit mit der göttlichen Natur an sich betrachtet unendlich. Sie gilt in dieser Hinsicht als unbestimmt (indeterminate), und alle möglichen Unterschiede sind in der Einheit des Unendlichen nicht zu unterscheiden (confuse). Für alle drei göttlichen Personen ist sie die gleiche Vernunft und die gleiche Vollkommenheit. In Gott gibt es keine Abstufungen der Vernünftigkeit. Aber in der Tätigkeit dieser Vernunft entstehen dennoch notwendig nähere Bestimmungen, die notiones determinantes, die die Personen als unterschiedliche Personen konstituieren. Der Vater ist die göttliche Natur als Zeugender des Sohnes und des Heiligen Geistes, ohne selbst gezeugt zu sein. Der Sohn ist die göttliche Natur als vom Vater Gezeugter und als Zeugender des Heiligen Geistes. Und der Heilige Geist ist die göttliche Natur als vom Vater und Sohn Hervorgebrachter, der selbst nicht hervorbringt $^{26}$.

Diese notwendigen Beziehungen beeinträchtigen die Einheit der göttlichen Natur nicht, denn es ist immer die gleiche lebendige Natur, die zeugt oder gezeugt wird. Deshalb ist jede der Personen mit der göttlichen Natur wesensgleich, oder wie es bei Albertus in Anlehnung an Boethius heißt: in Gott sind Ausdruck oder quod est (die Person) und Grund oder esse (die Natur) eins. Die Unterschiede bestehen nur zwischen den Formen des quod est, nicht im esse ${ }^{27}$. Sie sind Ausdruck der dreifachen Mitteilung der

Op. cit., q. 31, c. 2: S. 244b (5).

Op. cit., q. 30, c. 3, a. 1: S. 231a-235a; q. 41., c. 1, a. 2 + c. 2, a. 1: S. 315b-318b.

Op. cit., tr. 6 , q. 29 , c. 1 , a. 2 : S. 217 b-220b. 
einen göttlichen Natur. Sie bedingen sich gegenseitig in der Mitteilung dieser einen Natur an die drei Personen. In der höchsten Form der Vernünftigkeit gehen Einheit und Vielheit im Austausch der ungeteilten Natur zusammen. Der allgemein tätige Intellekt (intellectus universaliter agens) gestaltet sich in seiner Tätigkeit als Dreieinheit.

\subsection{Die Notwendigkeit der Dreiheit}

Der dritte Punkt, der uns hier interessiert, betrifft die besondere Form der Gestaltung dieser höchsten Form des Erkennens. Warum drei Personen?

Die Antwort des Thomas ist bekannt. Er deutete das Hervorgehen der Personen anhand der göttlichen Attribute Intellekt und Wille. Der Intellekt und der Wille stellen die beiden inneren Tätigkeiten der göttlichen Natur dar. Der Unterschied zwischen diesen immanenten Akten bestimmt auch den Unterschied zwischen den beiden innergöttlichen Hervorbringungen. Der Sohn wird nach der Art des Erkennens gezeugt, wohingegen der Heilige Geist aus einem Akt des Willens hervorgeht. Zusammen mit dem Vater, der als Prinzip und Urgrund dieser beiden Zeugungen gilt, gibt es also drei Personen in Gott $^{28}$. Dieses methodische Verfahren läßt sich auch bei anderen Autoren wie Heinrich von Gent feststellen ${ }^{29}$. Es hat sich jedoch nicht als feste Lehre bei den Dominikanern des späten dreizehnten und frühen vierzehnten Jahrhunderts durchsetzen können. Die bekanntesten Kritiker innerhalb des Ordens waren Jakob von Metz, Hervaeus Natalis und Durandus de Saint Pourçain ${ }^{30}$.

Albertus folgt einer anderen Spur. Er versucht, die Dreiheit der göttlichen Personen, die er wie Thomas als geoffenbarten Glaubenssatz annimmt, nicht so sehr psychologisch aus der Tätigkeit des göttlichen Geistes, sondern eher aus dem logischen Verständnis der Beziehungen selbst zu erklären ${ }^{31}$. Diese Methode paßt auch zum Aufbau seines Sentenzenkommentars sowie seiner Summa theologiae: die göttlichen Attribute werden erst nach der Trinität erörtert. Sie werden von Albertus eher aus der Perspektive der Schöpfung als im Hinblick auf die Deutung der Dreiheit der göttlichen Natur studiert.

Eine zentrale Rolle spielt bei Albertus der Begriff der natürlichen Ordnung (ordo naturae). Die Mitteilung der göttlichen Wesenheit wird durch nichts innerhalb oder außerhalb Gottes gehemmt. Sie gründet in der einzigen und absolut einfachen göttlichen Natur. Dennoch folgt sie einer natürlichen Logik, die zum Ausdruck bringt, daß der Vollzug der Mitteilung in den drei Personen seine höchste Vervollkommnung er-

THOMAS de Aquino: Summa theologiae, Ia, q. 27, a. 3 + a. 5.

Vgl. HENRICUS a Gandavo: Quodlibeta, Quodl. 5, q. 1: Paris 1518 (Nachdr. Louvain 1961), fol. $152^{\mathrm{r}} \mathrm{P}$.

Dazu COURTH [Anm. 14], S. 115-118.

ALBERTUS Magnus: Summa I, tr. 7, q. 31, c. 1: Ed. Colon. t. 34,1, S. 240b-242b; tr. 9, q. 42, c.

3: S. 328a-330b. 
reicht hat. Wenn die drei Personen hervorgebracht sind, hat die göttliche Mitteilung sich in alle möglichen Differenzierungen vollzogen und ist zum höchsten Abschluß gekommen $^{32}$.

Diese natürliche Logik entspricht einer abstrakten Gesetzmäßigkeit, die in jeder Mitteilung zum Ausdruck kommt und die bei Gott ihre absolute Form erreicht. Es gehört zu den Eigenschaften des höchsten Gutes, daß es sich im Hervorbringen mitteilt. Diese Mitteilung kann jedoch nicht ohne innere Ordnung sein. Würde das höchste Gut sich unbeschränkt an eine Vielzahl und nicht an eine Dreiheit von Personen mitteilen, so stünde das im Widerspruch zu seiner Vollkommenheit ${ }^{33}$.

In seinem Beweis stützt Albertus sich auf die göttliche Absolutheit. Gott ist Prinzip im absoluten Sinne. Er umfaßt alle möglichen Formen, die ein Prinzip zum Prinzip bestimmen. Er bringt anderes hervor, ohne selbst hervorgebracht zu sein. Diese Vollkommenheit kommt in der Person der Vaters zum Ausdruck. Die anderen möglichen Formen des Prinzips finden ihre höchste Gestaltung in den anderen beiden Personen. Im Sohn tritt das Prinzip als das hervor, was anderes hervorbringt, selbst jedoch auch hervorgebracht ist, und im Heiligen Geist als das, was gänzlich hervorgebracht ist, ohne selbst hervorzubringen ${ }^{34}$.

Alle möglichen Gliederungen, die dem Prinzip in seiner sich selbst mitteilenden Tätigkeit zukommen können, sind somit in Gott vertreten. Ausgangspunkt ist die Bestimmung des Prinzips, wie sie vom Vater ausgesagt wird: non ab alio, a quo aliud. Bei Sohn wird das non ab alio verneint und beim Heiligen Geist das non ab alio und das $a$ quo aliud. Mehr Differenzierungen kann es beim genannten Ausgangspunkt nicht geben. Die Vollkommenheit des göttlichen Prinzips findet somit ihren höchsten Ausdruck in der Dreiheit der Personen ${ }^{35}$.

Auch die Entfaltung anderer Vollkommenheiten ist immer dreifach geordnet. Jede Mitteilung setzt ein Hervorbringendes und ein Hervorgehendes voraus, die in einer gemeinsamen Natur aufeinander bezogen sind. Das gilt sowohl für die natürliche als auch für die willentliche Mitteilung ${ }^{36}$. Anders als Thomas spricht Albertus nicht vom Intellekt und Wille als den Grundformen des Hervorbringens, sondern von Natur und Wille. Im Zeugen des Sohnes teilt der Vater ohne Vorbehalt die ganze göttliche Natur

Op. cit., tr. 9, q. 41, c. 2: S. 316a-323b; q. 42, c. 3: S. 328a-330b.

Vgl. den Text in der nächsten Anm. Siehe auch op. cit., tr. 9, q. 42, c. 3, sol.: S. 328, 88 f.: „Si enim attenditur ordo naturae, perfectio in tribus est ..."

Summa I, tr. 7, q. 31, c. 1, ad 1: Ed. Colon. t. 34,1, S. 242, 55-61: „,.. diffusio in multa propter naturae est imperfectionem; sed diffusio in tot quot exigit 'ordo naturae, quo alter est ex altero, non quo alter est prior altero', est naturae perfectae. Et hoc ordo ulterius non potest multiplicari formaliter nisi in tres, quorum unus sit non ab alio per negationem principii, alius qui ab alio a quo alius, et alius qui ab aliis a quo nullus“.

Ebd.: S. 242, 69-71: „Nec sunt plures differentiae positae in ratione principii; et ideo pluribus modis non potest multiplicari formali multiplicatione“.

Vgl. op. cit., tr. 9, q. 42, c. 3: S. 328a-330b. 
dem Gezeugten mit. Im Hervorbringen des Heiligen Geistes teilen Vater und Sohn wie aus einem Prinzip die gleiche göttliche Natur dem von beiden Hervorgebrachten mit. Diese letzte Mitteilung manifestiert sich in dem einzigen Willen, alles, was Vater und Sohn der Natur nach sind, dem Heiligen Geist mitzuteilen. Die Mitteilung ist absolut. Sie ist die höchste Form der Liebe. Aber sowohl das Erzeugen des Sohnes als auch das Hervorbringen des Heiligen Geistes sind beide in der gleichen Natur begründet: das Erzeugen in der Natur als Natur, das Hervorbringen in der Natur als Wille ${ }^{37}$.

In Bezug auf die göttliche Natur, die das absolut Ungeteilte und Einfache darstellt, muß nach Albertus angenommen werden, daß die Art des Hervorbringens sich bei dem Sohn und dem Heiligen Geist notwendig unterscheidet, obwohl die Natur identisch bleibt. Denn die absolute Einfachheit ist in ihrer Tätigkeit zugleich Dreiheit. Deshalb umfaßt die höchste Natur Gegensätze, die in jeder anderen Natur nicht zusammen auftreten können. Was bei den zusammengesetzten Naturen unmöglich ist, ist bei dem in seiner absoluten Identität verharrenden Wesen notwendig. Seine Natur wird auf zwei verschiedene Weisen innerlich hervorgebracht: ,... in nulla natura composita potest hoc esse, sed in natura, quae est in fine simplicitatis, necesse est, quod ita sit ${ }^{\text {(38 }}$.

Die Trinität ist somit ein Geheimnis des Glaubens, das sich in der verstandesmäßigen Betrachtung der göttlichen Natur, wie sie sich in der absoluten Vollkommenheit als personale Vernunft denkt, dem Menschen als ,natürlich' offenbart: In Gott sind Einheit und Vielheit der Natur nach eins.

\section{Ausblick}

In der obigen Darlegung sind natürlich nicht alle Aspekte der Trinitätslehre Alberts angesprochen. Die Untersuchung zielte darauf ab darzustellen, wie bei Albertus das Geheimnis der Trinität als Glaubensmysterium anerkannt wird, zugleich aber auch als höchste Form der Vernünftigkeit gilt.

Diese Spur wurde im fünfzehnten Jahrhundert wieder aufgegriffen, oft auch unter Verweis auf Albertus. Aber wo bei ihm die Vernunft dem Glauben folgt, ist die Situation im ausgehenden Mittelalter anders. Die Trinität wurde als ein metaphysisches Thema angesehen, das die intellektuelle Dynamik des ersten Prinzips zum Ausdruck bringt. Der Glaube war nicht mehr Ausgangspunkt, wie bei Albertus, sondern galt als Bestätigung der Metaphysik. Dennoch wurde die spekulative Betrachtung des ersten Prinzips nach wie vor als ein affektives Streben des Menschen verstanden, das in der Unendlichkeit des Ersten seinen Ursprung hat. 
Diese Tradition gewann eine deutliche Gestalt in den Werken des Albertisten Heymericus de Campo. Bei ihm nahm die Trinität als Symbol der höchsten Form der Vernünftigkeit und als erstes Prinzip alles Möglichen einen bedeutenden Platz ein. An anderer Stelle haben wir die Trinitätslehre des Heymericus und ihre Bezüge zu den Betrachtungen bei Albertus bereits ausführlich untersucht ${ }^{39}$. Noch näher zu untersuchen sind die Ausführungen in seinem Sentenzenkommentar ${ }^{40}$.

Noch deutlicher tritt der Ansatz Alberts in der Docta ignorantia des Cusanus hervor, der wohl durch Heymericus de Campo mit Albertus vertraut gemacht wurde. Das Absolute ist notwendig die allerhöchste Dreifaltigkeit. Die absolute Einheit zeugt die Gleichheit der Einheit, und aus der Einheit und der Gleichheit geht die Einheit der Einheit oder die Verbindung zutage. Die Dreieinheit des Absoluten tritt in den verschiedenen Formen des Seins, die alle vom Ersten abhängig sind, in wechselnder Gestalt hervor $^{41}$. Die Argumente des Cusanus stützen sich nicht auf die Offenbarung: die Schrift bestätigt vielmehr, was der Philosoph in einer rein metaphysischen Betrachtung über die Struktur der Wirklichkeit entdeckt hat ${ }^{42}$. Die von Cusanus in diesem Zusammenhang angewandte Regel besagt, daß, wo immer es ein Mehr oder Weniger gibt, man noch nicht zum Absoluten gelangt ist, sondern sich im Bereich des Geschöpflichen befindet. Nur dort, wo keine Hinzufügung mehr möglich ist, ist das Absolute gegeben. Im Absoluten fallen die Gegensätze zusammen. Die Einheit ist ebenso absolut wie die Dreiheit. Die Dreieinheit ist Urbild des Zusammenfallens der Gegensätze ${ }^{43}$.

Bei Albertus gehen Vernünftigkeit und Glaube zusammen. Er spricht von der Theologie als scientia affectiva. Ähnliches gilt für die Metaphysik des Cusanus, wenn er vom Erstaunen beim Erkennen des ersten Prinzips spricht: eine Erkenntnis, die zugleich

HOENEN, Maarten J.F.M.: Trinität und Sein. Der Traktat ,De signis notionalibus trinitatis et unitatis supernae' und seine Bedeutung für das trinitarische Weltbild des Heymericus de Campo. In: Freiburger Zeitschrift Für Philosophie und Theologie 45 (1998), S. 207-263.

Der Kommentar ist in der Handschrift Bernkastel-Kues, St. Nikolaus-Hospital, Cod. Cus. 106, fol. $13 r-22 r$ erhalten. Eine Edition ist in Vorbereitung.

Noch immer grundlegend zur Trinitätslehre des Nicolaus de Cusa ist HAUBST, Rudolf: Das Bild des Einen und Dreieinen Gottes in der Welt nach Nikolaus von Kues. Trier 1952 (Trierer Theologische Studien, 4). Dort wird auch auf die Bezüge zwischen Heymericus de Campo und Cusanus eingegangen.

Dazu mit Angaben zur weiteren Literatur HOENEN, Maarten J. F. M.: Tradition and Renewal: The Philosophical Setting of Fifteenth-Century Christology. Heymericus de Campo, Nicolaus Cusanus, and the Cologne Quaestiones vacantiales (1465). In: EMERY, Kent / WAWRYKOW, Joseph (Hrsg.): Christ among the Medieval Dominicans: Representations of Christ in the Texts and Images of the Order of Preachers. Notre Dame, Ind. 1998, S. 462-492. 
Wissen und Nicht-Wissen einschließt und die den Menschen zum Absoluten hinzieht. Der Mensch erkennt das Absolute in seinem Drang, es erkennen zu wollen. Die Hingabe geht dem Erkennen voraus, ähnlich wie der Glaube dem Wissen ${ }^{44}$.

Die Trinitätslehre des Albertus belegt auf eindringliche Weise die Suche nach einer rationalen Begründung des Glaubens in einer geschichtlichen Periode, die gekennzeichnet ist von der Überzeugung, daß der Mensch in seinem Denken den Weg zu Gott finden kann. Heymericus und Cusanus teilen diese Überzeugung und betrachten wie Albertus die Trinität als höchste und absolute Form des Denkens. Dieser Gedanke als solcher ist nicht neu. Seine Quellen gehen zurück bis in die Antike. Er gehört zu den wichtigsten Themen der Metaphysik von Pythagoras und Aristoteles bis Schelling und Hegel. Aber er hat im Werk des Albertus eine für das Mittelalter bedeutsame Gestalt gewonnen, die am Vorabend der Neuzeit bei Heymericus de Campo und Nicolaus Cusanus das Denken über die göttliche Natur und über das menschliche Erkennen dieser Natur geprägt hat. 